\title{
Del nuevo producto biológico para el cáncer al impacto en la salud poblacional
}

\author{
From the new biologic cancer drug to the impact in population \\ health
}

\author{
Dr. C. Agustín Lage Dávila, Dr. C. Tania Crombet Ramos \\ Centro de Inmunología Molecular. La Habana, Cuba
}

\section{RESUMEN}

La investigación clínica no termina con el registro del nuevo medicamento, sino con la modificación de los indicadores de salud poblacional. Este artículo ilustra la experiencia de la biotecnología cubana en el propósito de contribuir a la reducción de la mortalidad por tumores malignos. En el control del cáncer, la biotecnología tiene cuatro espacios de impacto: el primero es en la prevención primaria mediante vacunas profilácticas, el segundo, consiste en las técnicas de diagnóstico precoz, el tercero es la estratificación de los pacientes mediante marcadores moleculares pronósticos o predictivos y el cuarto rol radica en el tratamiento de la enfermedad diseminada con vacunas terapéuticas y anticuerpos monoclonales que reconocen blancos específicos en los tumores. El uso de estas terapias ha traído consigo un cambio de paradigma del tratamiento del cáncer. La experiencia cubana ha sido exitosa debido a la existencia de un sistema de salud con altos estándares basado en la cobertura completa y equidad de acceso, el desarrollo del nivel primario de atención médica, centro de gravedad del sistema cubano y el desarrollo en las últimas tres décadas de una industria biotecnológica nacional, innovadora y con capacidad productiva para cubrir las necesidades nacionales de productos y exportar. A pesar de los avances, quedan grandes retos técnicos y metodológicos. La investigación científica posregistro permitirá trazar la estrategia para optimizar el impacto de los nuevos productos en la salud poblacional, encaminada a incrementar la esperanza de vida de los cubanos.

Palabras clave: biotecnología, cáncer, vacunas, anticuerpos monoclonales, nimotuzumab, CIMAvax EGF. 


\section{ABSTRACT}

The clinical research does not finish with the registration of a new drug, but with the modification of the population health indexes. This paper set forth the experience of the Cuban biotechnology in cancer control, in order to achieve the reduction of mortality rate from malignant tumors. Biotechnology has four areas of remarkable impact in cancer control: first, the primary prevention by means of prophylactic vaccines; second, the techniques for early diagnosis; third, the stratification of patients according to the molecular prognostic or predictive biomarkers and fourth, the treatment of advanced disseminated cancer with therapeutic cancer vaccines and monoclonal antibodies that detect specific targets in tumors. The use of these therapies has entailed a shift of paradigm in the cancer therapy. The Cuban experience has been successful due to the existence of a high standard health system based on complete coverage and equal access, the development of the primary medical care- the core of the Cuban health system - and the advance of a national, innovative biotech industry with manufacturing capacity to meet the national demand and to export products. In spite of the advances, big technical and methodological challenges still remain. Post-registration scientific research would define the strategy to optimize the impact of the new products in the population's health, aimed at increasing the life expectancy of the Cuban people.

Key words: biotechnology, cancer, vaccines, monoclonal antibodies, nimotuzumab, CIMAvax EGF.

\section{NTRODUCCI ÓN}

\section{La ciencia más allá de los productos}

Frecuentemente se identifica a la biotecnología como la obtención de nuevos productos. Este artículo no es sobre eso, sino sobre la actividad científica que debe ocurrir después de que un nuevo producto es registrado, para maximizar el impacto de este producto en la salud humana a escala poblacional. En el sesgo que tiene la literatura sobre la biotecnología en el trayecto entre la idea científica y el producto registrado, se oculta la creencia (o la propuesta intencionada) de que una vez realizados los ensayos clínicos que prueben ventaja estadísticamente significativa para el nuevo producto, el impacto de este en indicadores de salud ocurrirá automáticamente, mediante una buena labor de divulgación.

La historia de la salud pública demuestra que esto no es verdad. Entre la prueba científica de la vacunación antiviruela (1798) y la erradicación de la enfermedad transcurrieron 200 años, ${ }^{1}$ entre la vacuna de la poliomielitis (1955) y la eliminación de la enfermedad en el continente americano (1994) pasaron 40 años. ${ }^{2}$ También hubo otros 40 años entre la aparición de las primeras drogas antitumorales citotóxicas y la posibilidad de curación de la leucemia linfoblástica. ${ }^{3}$

La traducción de un nuevo producto en salud poblacional no es un proceso rápido. Tampoco es espontáneo: la optimización de los esquemas terapéuticos, la combinación del nuevo producto con las tecnologías previamente existentes, la identificación de los efectos a largo plazo, el perfeccionamiento de los procesos productivos para reducir los costos y hacer el nuevo producto accesible, y finalmente, 
la inserción de la nueva tecnología en las políticas de salud, todo ello, requiere generalmente nueva investigación científica. Requiere además nuevas metodologías de la investigación científica, más allá del reduccionismo y las condiciones controladas "de laboratorio" propias de la investigación prerregistro.

Este tipo de ciencia, encaminada a obtener los conocimientos necesarios para maximizar el impacto en salud de un nuevo producto, recibe mucha menos atención y recursos que la ciencia encaminada a obtener el producto. Ello se debe en gran medida a que el financiamiento de la investigación médica mundial proviene de manera creciente de la industria farmacéutica privada. ${ }^{4}$

La fracción de ensayos clínicos financiada por la industria privada pasó de menos del $5 \%$ en el periodo 1975-1984, a más del $55 \%$ en el periodo 1995-2004 y sobrepasa hoy el $70 \% .{ }^{5}$ La estrategia de la industria privada, una vez obtenido el registro de un nuevo producto, se concentra en el marketing; y el impacto del nuevo producto en la salud poblacional raramente se investiga.

\section{LOS ESPACI OS DE LA BI OTECNOLOGÍ A EN EL CONTROL DEL CÁNCER}

La industria biotecnológica surge a finales de los 70, a partir de la capacidad de clonar genes y expresarlos en una célula hospedero, la cual puede cultivarse a gran escala en fermentadores. Esta tecnología creó la capacidad de producir virtualmente cualquier molécula biológica en escala industrial y con la misma pureza y reproducibilidad de los fármacos químicos. De pronto el espacio de diversidad de los fármacos se ensanchó pues en principio cualquiera de los productos de nuestros 35000 genes o los 1000 millones de anticuerpos diferentes que tenemos, pudiera transformarse en un fármaco con posibilidades de industrializarse. Pero no se trata solo de mayor cantidad de candidatos a fármaco, sino también de fármacos diferentes, especialmente por el tamaño de las moléculas. Un anticuerpo, con un peso molecular de $150000 \mathrm{Da}$, tiene un tamaño cientos de veces superior al de los fármacos químicos; y el tamaño molecular influye mucho en la especificidad de las interacciones moleculares. De ahí la promesa de drogas de acción más específica.

Esta opción dio lugar al surgimiento de una industria que hoy comprende unas 5000 empresas en el mundo, y a una intensa actividad de investigación desarrollo, que se expresa en más de 900 fármacos y vacunas en ensayo clínico. ${ }^{5}$ Las aplicaciones potenciales son enormes, pero en el orden práctico dos campos de aplicación han dominado el panorama: las vacunas y el cáncer.

En el control del cáncer se dibujan cuatro espacios posibles de impacto de la biotecnología: el primero, es la prevención primaria mediante vacunas, pues probablemente hasta el $30 \%$ de las neoplasias está vinculado con infecciones. Las vacunas para los virus de hepatitis B y papiloma, pueden reducir la incidencia primaria de hepatocarcinoma y cáncer de cérvix respectivamente. ${ }^{6}$ El segundo espacio, es el diagnóstico precoz, mediante el desarrollo de métodos de detección de antígenos asociados a tumores (como el Antígeno Prostático Específico) o concentrando la pesquisa en grupos de riesgo identificados mediante marcadores genéticos. ${ }^{7}$ El tercer espacio, es el de la estratificación de los pacientes y la identificación mediante marcadores moleculares de subgrupos con diferente pronóstico o diferente sensibilidad a los tratamientos, lo que es el camino hacia la medicina personalizada. ${ }^{8}$ Finalmente, está el espacio del tratamiento de la enfermedad diseminada, estadio al que llega más del $50 \%$ de todos los casos diagnosticados. En esta etapa, una vez más la biotecnología promete no solamente 
nuevas drogas, ${ }^{9}$ sino cambios esenciales en la estrategia terapéutica, que son principalmente tres:

1. La transformación del cáncer avanzado en una enfermedad crónica, ${ }^{10}$ al disponerse de fármacos poco tóxicos que pueden utilizarse mucho tiempo, extendiendo la supervivencia y conservando la calidad de vida.

2. La posibilidad de tratamientos oncoespecificos en pacientes ancianos o con comorbilidades que usualmente no son elegibles para las terapéuticas agresivas de hoy. ${ }^{11}$

3. La posibilidad de tratamientos oncoespecificos en el nivel primario de atención medica, lo que es un corolario del concepto de cronicidad.

\section{COMPLEJ I DADES REALES Y OBSTACULOS CONSTRUI DOS}

Producir impacto en salud poblacional es un objetivo mucho más difícil que obtener un nuevo producto. Exige, ya no solo obtener diferencia estadísticamente significativa en un ensayo clínico, sino ser capaces de modificar "indicadores duros y objetivos" de incidencia, mortalidad y supervivencia en poblaciones no seleccionadas.

El reto es muy complejo: las intervenciones terapéuticas incluyen varias tecnologías y productos que funcionan "en bloque", donde es difícil identificar el aporte de cada componente, la población de pacientes es heterogénea y lo será cada vez más en la medida que tengamos más pruebas de laboratorio con poder de estratificación; y los efectos ocurren a largo plazo, especialmente en tratamientos crónicos. Para obtener evidencia científica que guíe decisiones en tales condiciones, las actuales metodologías de ensayo clínico no son suficientes, pues estas se han diseñado para evaluar componentes aislados de una intervención salubrista, en poblaciones homogéneas, y en condiciones controladas. Hay que seguir "haciendo ciencia" después.

Otro desafío está en el costo de los procesos productivos. La biotecnología, como toda tecnología emergente, transita por una etapa de adolescencia industrial, en la cual los costos de producción son altos. Ello es especialmente así en el caso de los anticuerpos monoclonales terapéuticos, cuyos procesos no se pueden basar en bacterias y levaduras, sino que exigen fermentación de células de mamíferos. ${ }^{12}$ Se requerirá mucho esfuerzo científico y tecnológico para reducir costos, tal como ha ocurrido por ejemplo, en la industria de las computadoras.

Pero además de estas complejidades enraizadas en las características de las enfermedades, los productos y los procesos; el trayecto hacia el impacto en salud poblacional contiene obstáculos artificiales, derivados de la estructura socioeconómica de la industria farmacéutica y los sistemas de salud en los países industrializados que controlan hoy la economía mundial. Estos obstáculos se expresan en la manera en que se conforman los precios de los medicamentos y en las fuentes de financiamiento de la investigación científica.

La industria farmacéutica mundial está dirigida por "las fuerzas del mercado", y esto tiene dos implicaciones: que los precios se fijan por la "demanda solvente" (el que puede pagar) y no por la demanda real; y que la estrategia competitiva de las empresas se basa en la "construcción de barreras de entrada" que impidan el acceso 
al mercado de nuevos competidores. Estas barreras de entrada han sido esencialmente dos: la barrera de las patentes y la barrera de las regulaciones.

El impacto de la propiedad intelectual sobre los medicamentos es un fenómeno reciente, impulsado por los Acuerdos de Propiedad Intelectual TRIPS que surgieron con la Organización Mundial del Comercio en $1995 .{ }^{13}$ Se trata de una forma de privatización del conocimiento: la exclusividad comercial que conceden las patentes permite al propietario establecer precios monopólicos durante cierto tiempo. Este plazo teóricamente debería limitarse a 20 años, después de lo cual deben aparecer copias genéricas baratas del producto. Sin embargo ahora, al expirar las patentes de los primeros productos biotecnológicos, se plantea la polémica de cuan idénticas deben ser dos moléculas biológicas para que una sea admitida como copia genérica de la otra. En el momento que se escribe este artículo aún está abierto el debate ante la peligrosísima tesis de que dos moléculas biológicas nunca son exactamente iguales, y entonces todo "biosimilar" debe repetir la investigación clínica, lo que equivaldría en la práctica, a limitar el surgimiento de biológicos de menor costo, y a otorgar a los primeros productores una "patente para siempre."14

Esta polémica ocurre precisamente en el momento en que los productos biológicos ocupan un espacio creciente en la farmacopea, que se prevé para el 2016 como superior al $20 \%$ para todos los fármacos y cercano al $50 \%$ para los 100 fármacos de más ventas. ${ }^{5}$ Se podría imponer una farmacopea biotecnológica con productos artificialmente costosos.

El enfoque de mercado de la industria farmacéutica mundial tiene además otra consecuencia para el impacto de los productos en la salud poblacional, y es que es hoy la propia industria farmacéutica la que financia en más del $70 \%$ la investigación médica. Al hacerlo define aquello que se investiga y lo que no se investiga. No es extraño entonces que el posicionamiento de un nuevo producto en las políticas de salud resulte guiado por acciones de comercialización, más que por un enfoque científico de su rol en el control poblacional de las enfermedades.

\section{LA OPORTUNI DAD CUBANA}

En Cuba coinciden tres circunstancias que conforman un espacio de oportunidad para la proyección de la biotecnología sobre la salud poblacional:

1. la existencia de un sistema de salud que ha sido exitoso durante décadas en lograr altos estándares de salud poblacional aun con escasos recursos, basado en los principios de la cobertura completa, la equidad de acceso y en la evaluación sistemática de los indicadores,

2. el desarrollo del nivel primario de atención médica, centro de gravedad del sistema cubano, que se apoya en su capital humano, de ahí que Cuba sea actualmente el país del mundo con mayor cantidad de médicos por habitante, y

3. el desarrollo en las últimas tres décadas de una industria biotecnológica nacional, innovadora, y con capacidad productiva para cubrir las necesidades nacionales de productos y exportar.

Estos tres factores no se dan simultáneamente en tal magnitud, en ningún otro país. 
Es ciertamente una oportunidad. Pero aprovecharla bien depende mucho de como utilizamos estas capacidades, y ello de nuevo, es un problema científico.

En el caso concreto del control del cáncer, la oportunidad está dada hoy por la existencia en la industria biotecnológica cubana de más de 25 proyectos de productos de uso oncológico que incluyen sistemas de diagnóstico, moléculas biosimilares como la eritropoyetina recombinante, el factor estimulante de colonias granulocíticas, los anticuerpos monoclonales, junto con varios anticuerpos monoclonales y vacunas de cáncer patentados por instituciones cubanas.

Los productos que ya están registrados (recuadro) porque ya produjeron beneficios terapéuticos estadísticamente significativos en un ensayo clínico, pudieran producir un impacto mayor si investigamos formas mejores de usarlos y de combinarlos con otros tratamientos.

Recuadro. Productos para el cánoer en desarrollo por las instituciones del polo científico del oeste de La Habana

\begin{tabular}{|c|c|c|}
\hline Evaluación preclínica & En ensayo clínico & Productos registrados \\
\hline Anticuemo 14F7 & Anticuerpo anti CD20 & Interferón \\
\hline Anticuerpo anti Her2 & Vacuna Her 1 & $\begin{array}{l}\text { Factor estimulante } \\
\text { de oolonias }\end{array}$ \\
\hline Vacuna Pan Her & Vacuna gangliósidos & Eritropoyetina \\
\hline Muteinas IL2 & Péptido CIGB 300 & Anticuerpo nimotuzumab \\
\hline Muteína TGF alfa & Vacuna C. próstata & Vacuna CimaVax EGF \\
\hline Vacuna terapéutica HPV & Heberferón & Vacuna racotumomab \\
\hline Vacuna VEGF & & \\
\hline Péptido VEGF & & \\
\hline Péptido CIGB 552 & & \\
\hline \multicolumn{3}{|c|}{ Diagnóstico } \\
\hline \multirow[t]{3}{*}{ Mamógrafo digital } & & Kit de citología de cuello \\
\hline & & Sistema de PSA \\
\hline & & $\begin{array}{l}\text { Sistema de sangre oculta } \\
\text { en heces fecales }\end{array}$ \\
\hline
\end{tabular}

En general, las terapias "dirigidas a blancos específicos", dentro de las cuales se incluyen los anticuerpos monoclonales y las vacunas de cáncer, permiten una estrategia diferente de atención al paciente con cáncer avanzado. Al reconocer dianas o blancos específicos en los tumores, se pueden administrar de forma prolongada en pacientes añosos o con comorbilidades y en combinación con otros tratamientos. ${ }^{15}$

El anticuerpo monoclonal humanizado nimotuzumab reconoce el receptor del factor de crecimiento epidérmico (EGFR), el cual está sobrexpresado en muchos tumores de origen epitelial, con la inclusión de neoplasias de pulmón, próstata, cabeza y cuello, sistema digestivo, cuello de útero y otras. La racionalidad científica del uso prolongado del monoclonal nimotuzumab, se sustenta en la necesidad de inhibir la señalización del EGFR, durante toda la historia natural de enfermedad. ${ }^{16}$ Además del efecto en la inhibición de la transmisión de las señales oncogénicas, los anticuerpos pueden estimular la citotoxidad mediada por células de la inmunidad natural y por complemento. ${ }^{17}$ 
En los ensayos iniciales, los esquemas terapéuticos preveían el uso de hasta seis dosis del nimotuzumab. La práctica médica subsecuente demostró que había beneficio clínico, sobre todo en la supervivencia, si el paciente seguía recibiendo tratamiento con el anticuerpo.

Una revisión sistemática de cinco ensayos diferentes en cáncer avanzado de cabeza y cuello, detectó ventaja significativa de supervivencia para los pacientes en que el tratamiento fue por más de seis dosis, comparado con cuatro ensayos, donde el esquema de nimotuzumab consistió en solo 6 ciclos (Fig.).

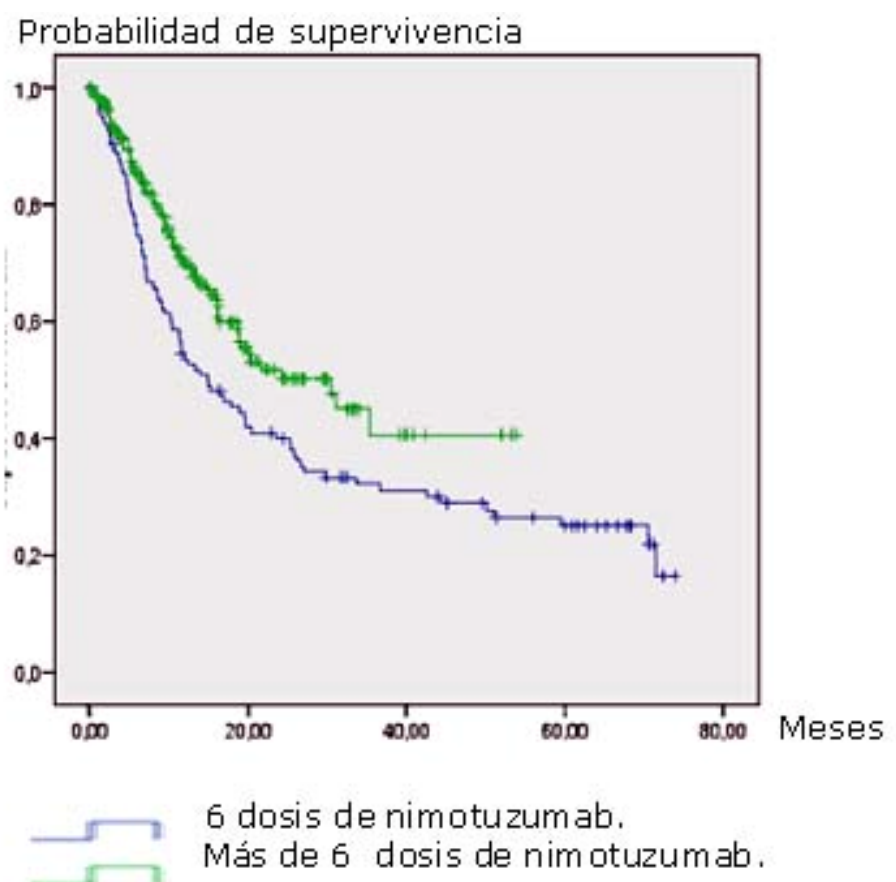

Fig. Uso del nimotuzumab en pacientes con tumores de cabeza y cuello: 6 dosis vs. tratamiento crónico (más de 6 dosis). Incremento significativo de la supervivencia de los pacientes que reciben tratamiento prolongado con el anticuerpo monoclonal nimotuzumab.

A pesar de la administración prolongada, no hay evidencias de toxicidad acumulativa, incluso en pacientes que han recibido más de 100 dosis del anticuerpo.

El concepto de tratamiento de larga duración es una de las prioridades actuales de la investigación clínica con este producto.

También en el caso de las vacunas terapéuticas las inmunizaciones prolongadas son necesarias para la inducción de una respuesta inmune potente. La vacuna CIMAvax EGF, es un conjugado inmunogénico del factor de crecimiento epidérmico (EGF) humano recombinante con la proteína p64 de la Neisseria meningitides. Induce una respuesta de anticuerpos contra el EGF propio, que es uno de los factores de crecimiento importantes en las neoplasias de pulmón. ${ }^{18}$

La vacunación prolongada ha sido muy segura, y los esquemas terapéuticos que se están investigando hoy mantienen la vacunación mensual durante tiempo prolongado, después de la quimioterapia. El tiempo de tratamiento más largo ha sido de diez años 
en cuatro pacientes del ensayo clínico fase II, y no hay evidencia de toxicidad acumulativa, ni de agotamiento de la respuesta inmune. La vacunación crónica incrementa el porcentaje de pacientes clasificados como altos respondedores y existe una correlación significativa entre la buena respuesta inmune y la supervivencia. ${ }^{19,20}$

Otra dirección importante de investigación es la del uso de los anticuerpos y vacunas en pacientes añosos o portadores de comorbilidades. Dada la escasa toxicidad de los productos, los pacientes portadores de enfermedades crónicas compensadas, no se excluyen de los ensayos clínicos y se han diseñado ensayos clínicos especialmente en pacientes no aptos para recibir quimio o radioterapia.

La tercera consecuencia de la emergencia de la inmunoterapia, es la posibilidad de desplazar el centro de tratamiento, de la atención secundaria a la atención primaria de salud. La administración de estas terapias en los consultorios o policlínicos garantiza un mayor acceso de los pacientes a las drogas y un mejor cumplimiento del programa terapéutico. El Centro de Inmunología Molecular (CIM) conduce un ensayo fase IV con la vacuna CIMAvax EGF, en pacientes en la atención primaria de salud. En tres años se han tratado más de 700 pacientes en 65 policlínicos de todas las provincias.

Los datos del ensayo clínico fase IV en los policlínicos han confirmado el perfil de seguridad y eficacia de la vacuna obtenidos en los ensayos fase III.

Existe otra oportunidad de aumento del impacto de los nuevos productos en la salud dada la posibilidad de administrar tratamientos combinados. El anticuerpo nimotuzumab se usa en combinación con las terapias oncoespecificas, actuando como quimio o radiosensibilizador. En los tumores de cabeza y cuello y de esófago, la combinación con radioterapia duplica la tasa de respuesta completa antitumoral. En los astrocitomas de alto grado de malignidad, se duplica la supervivencia de los pacientes cuando el nimotuzumab se usa concurrentemente con dosis curativas de irradiación. Los datos iniciales de los ensayos de combinaciones indican que la inmunogenicidad de las vacunas no se afecta por la quimioterapia.

\section{HACI A UNA AGENDA CI ENTÍ FI CA ORI ENTADA A LA SALUD POBLACI ONAL}

La investigación científica orientada al impacto poblacional comienza cuando se tiene un producto registrado. Vamos a discutir el tema a partir de los productos concretos de la biotecnología cubana ya registrados o previstos a registrar en los próximos dos años. Estos son:

- Dos sistemas de diagnóstico (antígeno prostático y para sangre oculta en heces fecales).

- Dos citocinas (el interferón alfa y el factor estimulador de colonias granulociticas).

- Tres anticuerpos monoclonales (el nimotuzumab anti-EGFR y los biosimilares rituximab y tratuzumab).

- Dos vacunas terapéuticas (la vacuna anti EGF CIMAVAX y la vacuna idiotipica racotumomab). 


\section{¿Qué nuevos conocimientos necesitamos para maximizar el impacto de estos productos en el control del cáncer a escala poblacional?}

Esencialmente estos:

1. La eficacia del diagnóstico precoz en poblaciones de riesgo. En la literatura extranjera hay consenso sobre la capacidad de la prueba de sangre oculta en heces fecales para reducir mortalidad por cáncer de colon. ${ }^{21}$ La literatura sobre el uso masivo del antígeno prostático específico, en cáncer de próstata todavía es contradictoria. ${ }^{22}$ No obstante en ambos casos la eficacia se puede multiplicar concentrando el tamizaje en los grupos de riesgo, entre ellos la agrupación familiar de la cual ya tenemos datos en Cuba. El valor predictivo de la positividad de una prueba diagnóstica depende de la estrategia de uso, de la prevalencia de la enfermedad y del riesgo relativo de los grupos a los que se aplique.

2. La calidad en el proceso, la utilización de los productos. Los nuevos productos crean un nuevo patrón de tratamiento óptimo, pero la práctica médica real frecuentemente se desvía de ese óptimo de calidad por innumerables razones. Necesitamos investigaciones operacionales que caractericen y cuantifiquen las desviaciones del patrón óptimo de atención en nuestras condiciones concretas, e identifiquen las intervenciones necesarias para corregirlas.

3. El comportamiento de los productos novedosos en poblaciones no seleccionadas. La prueba de concepto de un nuevo producto se obtiene mediante ensayos clínicos en poblaciones seleccionadas. Luego es imprescindible reevaluar el desempeño de los productos cuando se aplican a toda la población de pacientes, y recalcular en esas condiciones las cifras de respuesta, supervivencia y toxicidades. Son los objetivos del ensayo clínico fase IV de los cuales ya tenemos varios en marcha.

4. El espectro de actividad de los productos novedosos en otras localizaciones tumorales. El anticuerpo monoclonal nimotuzumab tiene datos de eficacia en tumores de cabeza y cuello, cerebro y esófago. Pero el "blanco" de este anticuerpo se encuentra en muchos tumores derivados de epitelios, que son el $70 \%$ de la oncología. Actualmente tenemos ensayos clínicos en curso para evaluar este producto en neoplasias de pulmón, páncreas, estómago y cuello de útero. Algo similar sucede con la vacuna CIMAvax EGF, hoy registrada para cáncer del pulmón, pero con un ensayo clínico en curso para cáncer de próstata hormono resistente.

5. La identificación de subgrupos de pacientes más sensibles al tratamiento. Una de las piezas de conocimiento más importantes es la identificación de aquellos subgrupos de pacientes que responden mejor. Para los productos biosimilares, esta información está disponible en la literatura; pero para los productos que tienen patente cubana, debemos obtenerla nosotros. La identificación de estos subgrupos maximiza el efecto y reduce costos. Actualmente tenemos evidencia inicial del valor predictivo de la expresión del receptor EGF para el efecto del anticuerpo nimotuzumab, y de la concentración de EGF sérico para el efecto de la vacuna CIMAvax EGF. Estas evidencias deben ser confirmadas.

6. La potenciación del efecto antitumoral en combinaciones con otros tratamientos. A medida que la cantidad de nuevos productos aumenta linealmente, la cantidad de combinaciones posibles aumenta exponencialmente, y resulta imposible evaluarlas todas por "ensayo y error". Se impone identificar prioridades en base al conocimiento que tengamos del mecanismo de acción de los productos. Por ejemplo, en el caso del nimotuzumab, las prioridades parecen estar en la optimización de la combinación con radioterapia en los tumores radiosensibles, y con los citostáticos que tienen acción

http://scielo.sld.cu 
sobre el receptor de EGF. Para las vacunas terapéuticas que probablemente terminan todas generando alguna forma de inmunidad celular, son especialmente atractivos los citostáticos que inducen apoptosis inmunogénica y pueden potenciar la presentación de nuevos antígenos. Otra opción es el control de la inflamación crónica. ${ }^{23} \mathrm{La}$ percepción del rol de la inflamación en los tumores se ha modificado en la última década, al identificarse mecanismos por los cuales esta puede facilitar el crecimiento del tumor, la angiogénesis y el escape a la inmunidad. Todavía no hay un estudio sistemático del espacio del control de la inflamación en las combinaciones terapéuticas en cáncer.

7. El efecto del tratamiento prolongado. La transformación del cáncer avanzado en una enfermedad crónica demandará tratamientos oncoespecíficos de larga duración. Los efectos a largo plazo y la optimización de la duración del tratamiento son informaciones que generalmente no están disponibles cuando se registra un nuevo producto. El antecedente no biotecnológico está en los antiestrógenos e inhibidores de aromatasa en el cáncer de mama, que comenzaron utilizándose por un año y ensayos clínicos posteriores indicaron la ventaja de su uso dos años, luego cinco años, y ahora se explora el tratamiento por diez años. ${ }^{24}$ Para los anticuerpos monoclonales hay evidencia en la literatura sobre el uso prolongado del trastuzumab, ${ }^{25}$ y el rituximab. ${ }^{26}$ El nimotuzumab se ha utilizado en casos seleccionados hasta cinco años sin señales de toxicidad acumulativa, y varios de los ensayos clínicos en curso prevén mantenimiento mensual hasta que el estado general del paciente lo permita. El hecho de que los anticuerpos anti EGFR, inducen inmunidad celular es otro argumento a favor del uso prolongado. Para la vacuna CIMAvax EGF, también hay series de pacientes con uso prolongado y sin evidencias de agotamiento de la respuesta inmune. ${ }^{19}$

8. El efecto en pacientes ancianos o con comorbilidades. Con los cambios demográficos y el control de factores de riesgo, la edad promedio del diagnóstico de cáncer se está incrementando y el pronóstico es que más del $70 \%$ de las neoplasias en el 2030 ocurrirá en pacientes mayores de 65 años. El $25 \%$ de los individuos entre 65 y 69 años y el $50 \%$ de los individuos entre 80 y 84 años tiene dos o más enfermedades crónicas. ${ }^{27}$ Estos grupos están muy poco representados en los ensayos clínicos. Se requerirá un esfuerzo intencional de investigación clínica de los nuevos productos en este tipo de pacientes.

El uso amplio de la inmunoterapia en pacientes de edad avanzada requiere una caracterización de la senescencia del sistema inmune en la población cubana y hay un proyecto en curso que ya comienza a producir los primeros datos. Este proceso, caracterizado por la contracción del repertorio de células $T$, la expansión del compartimento de linfocitos T activados y la inflamación crónica sistémica, ${ }^{28}$ ocurre a velocidades diferentes en distintas poblaciones humanas, en función de sus condiciones genéticas y ambientales, y de la carga antigénica crónica a que están expuestas.

9. La inserción de los nuevos productos en paquetes de Intervención Compleja en Salud. La inmunoterapia de cáncer como acción aislada es poco probable que logre cambios grandes de supervivencia o reducción de mortalidad. La inmunoterapia debe ser parte de una intervención sanitaria integral que incluya la prevención primaria, el diagnóstico oportuno, la pesquisa activa, el tratamiento oncológico según las normas establecidas, la inserción de vacunas o monoclonales en los paquetes de tratamiento, así como el manejo del dolor, la nutrición, el apoyo psicológico al paciente y a la familia, entre otras medidas de cuidados continuos. Las nuevas drogas de inmunoterapia registradas, deben ser incorporadas a las guías diagnósticas y terapéuticas. Uno de los desafíos científicos de los próximos años será el de evaluar científicamente "paquetes terapéuticos de intervención" con varios componentes. 


\section{¿Cuáles herramientas metodológicas necesitamos perfeccionar para obtener esos conocimientos?}

No será suficiente identificar los nuevos objetivos. Necesitamos consolidar el dominio de la metodología del ensayo clínico en todo el Sistema Nacional de Salud, pero además desarrollar nuevas herramientas tales como:

1. El análisis de supervivencia a escala poblacional, que nos permita monitorear en tiempo real, no solo las tasas de incidencia y mortalidad (que ya se registran) sino la evolución de las tasas de supervivencia por localización, que son más sensibles en el corto plazo a las innovaciones terapéuticas.

2. La evaluación observacional de cohortes de pacientes tratados con los nuevos productos fuera del marco de ensayos clínicos. La red informática del Sistema Nacional de Salud cubano, puede acercarnos al objetivo de que todo paciente tratado con un nuevo producto aporte información. Ello requerirá a su vez la implementación en el sistema de nuevas tecnologías de análisis de datos.

3. La detección de agregación familiar y otros factores de riesgo genético, deberán constituirse en componentes de la dispensarización, que nos permitan guiar las intervenciones (cuyos recursos siempre serán limitados) hacia los subgrupos poblacionales donde pueden dar un impacto mayor.

4. La evaluación de intervenciones complejas en salud. La más abarcadora de ellas es el mismo Programa Integral de Control del Cáncer que conduce el MINSAP. Pero este a su vez puede descomponerse en intervenciones susceptibles de caracterización por indicadores de proceso y de impacto, y análisis científico.

La mortalidad por cáncer continúa siendo el principal obstáculo al incremento de la esperanza de vida de la población cubana, más allá de la cifra de 78 años que hoy tenemos. Aunque el cáncer es la segunda causa de muerte (21 740 fallecidos en el 2011) muy cercana a las enfermedades cardiovasculares (22 178 en el 2011), la cifra de años de vida perdidos por cáncer $(17,9)$ es muy superior a la correspondiente a cardiovasculares $(10,5)$. Más aún, la tasa de mortalidad por enfermedades cardiovasculares hoy es casi idéntica a la de 1993, mientras que la tasa de mortalidad por cáncer ha aumentado el $50 \%$ en igual periodo.

Esta tendencia tenemos que revertirla y la biotecnología puede aportar mucho a esa tarea. Productos existen ya. Pero es imprescindible que la investigación científica continúe más allá del registro de los productos, y nos dibuje la estrategia para optimizar el impacto de los nuevos productos en la salud de la población.

\section{REFERENCI AS BI BLI OGRÁFICAS}

1. Fenner F. Smallpox: emergence, global spread, and eradication. Hist Philos Life Sci. 1993; 15(3): 397-420.

2. de Quadros CA. History and prospects for viral disease eradication. Med Microbiol Immunol. 2002;191(2):75-81.

3. Aur RJ .Childhood acute lymphocytic leukemia. Compr Ther. 1976;2(4):48-56.

http://scielo.sld.cu 
4. Brezis M.Big pharma and health care: unsolvable conflict of interests between private enterprise and public health. Isr J Psychiatry Relat Sci. 2008;45(2):83-9.

5. The annual 2012 Pharmaceutical Industry Profile; Pharmaceutical Research and Manufacturers of America, Pharmaceutical Industry Profile 2012. Washington, D. C.: PhRMA, April; 2012.

6. Chambers CV. Cancer vaccines. Prim Care. 2011;38(4): 703-15. Epub 2011 Sep 28.

7. Smith RA, Cokkinides V, Brawley OW. Cancer screening in the United States, 2009: a review of current American Cancer Society guidelines and issues in cancer screening. CA Cancer J Clin. 2009;59(1):27-41.

8. Lamlertthon W, Hayward MC, Hayes DN. Emerging technologies for improved stratification of cancer patients: a review of opportunities, challenges, and tools. Cancer J. 2011; 17(6): 451-64.

9. Scott AM, Wolchok JD, Old LJ. Antibody therapy of cancer. Nat Rev Cancer. $2012 ; 12(4): 278-87$.

10. Hoos A, Eggermont AM, J anetzki SJ. Improved endpoints for cancer immunotherapy trials. J Natl Cancer Inst. 2010; 102(18): 1388-97.

11. Baleydier F, Domenech C, Thomas X. Novel conventional therapies in oncohemathology.Bull Cancer. 2011;98(8):901-13.

12. Farid SS. Process economics of industrial monoclonal antibody manufacture. J Chromatogr B Analyt Technol Biomed Life Sci. 2007;848(1):8-18.

13. Faunce TA, Drahos P. Trade Related Aspects of Intellectual Property Rights (TRIPS) and the threat to patients: a plea for doctors to respond internationally. Med Law. 1998; 17(3): 299-310.

14. Morrow T Rules for evaluating biosimilars put applicants through hoops. Manag Care. 2012;21(3):56-7.

15. Dimitrov DS, Marks JD. Therapeutic antibodies: current state and future trends -is a paradigm change coming soon? Methods Mol Biol. 2009; 525:1-27.

16. Perez R, Moreno E, Garrido G, Crombet T. EGFR-targeting as a biological therapy: understanding nimotuzumab's clinical effects. Cancer. 2011;3:2014-31.

17. Garrido G, Rabasa A, Sánchez B. Induction of immunogenic apoptosis by blockade of epidermal growth factor receptor activation with a specific antibody. J I mmunol. $2011 ; 187(10): 4954-66$.

18. Rodríguez PC, Rodríguez G, González G. Clinical development and perspectives of CIMAvax EGF, Cuban vaccine for non-small-cell lung cancer therapy. MEDICC Rev. 2010; 12(1): 17-23.

19. Gonzalez G, Crombet T, Lage A. Chronic Vaccination with a Therapeutic EGFBased Cancer Vaccine: A Review of Patients Receiving Long Lasting Treatment. Curr Cancer Drug Targets. 2011;11(1): 103-10. 
20. Neninger E, García B, Crombet T. Combining an EGF-based Cancer Vaccine With Chemotherapy in Advanced Nonsmall Cell Lung Cancer. J Immunother. 2009;32:929.

21. New guidance for colorectal cancer screening. Lancet. 2012;379(9820): 978.

22. O'Shaughnessy M, Konety B, Warlick C. Prostate cancer screening: issues and controversies. Minn Med. 2010; 93(8):39-44.

23. Kundu JK, Surh YJ. Emerging avenues linking inflammation and cancer. Free Radic Biol Med. 2012; 52(9):2013-37.

24. Burstein HJ, Prestrud AA, Seidenfeld J. et al. American Society of Clinical Oncology clinical practice guideline: update on adjuvant endocrine therapy for women with hormone receptor-positive breast cancer. J Clin Oncol. 2010;28(23):3784-96. Epub $2010 \mathrm{Jul} 12$.

25. von Minckwitz G, Schwedler K, Schmidt M; GBG 26/BIG 03-05 study group and participating investigators. Trastuzumab beyond progression: overall survival analysis of the GBG 26/BIG 3-05 phase III study in HER2-positive breast cancer. Eur J Cancer. 2011;47(15):2273-81.

26. Michallet AS, Coiffier B, Salles G. Maintenance therapy in follicular lymphoma. Curr Opin Oncol. 2011;23(5):449-54.

27. Hayat MJ, Howlader N, Reichman ME. Cancer statistics, trends, and multiple primary cancer analyses from the Surveillance, Epidemiology, and End Results (SEER) Program. Oncologist. 2007; 12(1):20-37.

28. Peters T. Immunosenescence. Current status and molecular mechanisms [review]. Hautarzt. 2011;62(8): 598-606.

Agustín Lage Dávila. Centro de Inmunología Molecular. PO Box 16040. Habana 11600. La Habana, Cuba. Correos electrónicos: lage@cim.sld.cu, taniac@cim.sld.cu 